\title{
SEASONAL EFFECTS OBSERVED IN TIME \\ DETERMINATIONS AT SANTIAGO
}

\author{
F. NOËL \\ Departamento de Astronomia, Universidad de Chile, and \\ European Southern Observatory
}

\begin{abstract}
A comparison of the Universal Time and local latitude deduced from the Astrolabe observations since 1966 to 1970, with respect to similar results obtained from BIH datas, show significative annual differences, specially in time, of a stable pattern. The consistence of the latitude differences (Astrolabe - BIH) and latitude group corrections, computed by the chain method, prove that these differences could be explained by the influence of a systematic error in the FK 4 of $\Delta \delta \alpha$ type. On the other hand the inconsistence of the strong annual differences in time with the time group corrections, show that these differences can not be explained by catalogue systematic errors. The group corrections in time are compared with an investigation of the FK4 $\Delta \alpha_{\alpha}$ error based in the observations made with a transit instrument at Santiago.
\end{abstract}

The purpose of the present paper is to show some annual effects found in the results of the Astrolabe observations at Santiago, Chile (lat. = -33.4) (Noël, 1968; Anguita, and Noël, 1969), during the period 1966-1970. These effects appear specially in time. If we compare the UT1 deduced from the observations with the UT1 of BIH, the differences Astrolabe-BIH are systematically greater in winter than in summer.

The summarized differences in Latitude Astrolabe-BIH are plotted in Figure 1, where we can see also the curve of the group corrections in latitude. Each point has been obtained from the difference between a smoothed observed latitude and the normal latitude computed with the polar coordinates given by the BIH, interpolated for the corresponding epoch. The polar coordinates for 1966 and 1967 are reduced to the Conventional International Origin (CIO).

There is a special feature in the general pattern of the curves without group corrections, which is repeated every year: minima close to the extremes of the year. The fact that this effect is eliminated in the curves with group corrections, leads us to think that the observed feature could be explained by the influence of a systematic error in the FK4 of $\Delta \delta_{\alpha}$ type.

The group corrections were computed by means of the method of differences for consecutive groups observed in the same night during 1966 and for nonconsecutive groups during 1967-1968 and 1969-1970 (Table I and Figure 3). The magnitudes of the closing errors for 1966 and 1967-1968 are of the order to be expected with the old value of the aberration constant. A remarkably small closing error was obtained in 1969-70 when during the whole period the new value of the aberration constant was used.

Considering the consistency of the group corrections obtained in the three periods and in order to have a more homogeneous system in time and latitude, the corrections applied in the results presented in this paper are those based on the observations made during 1966. 

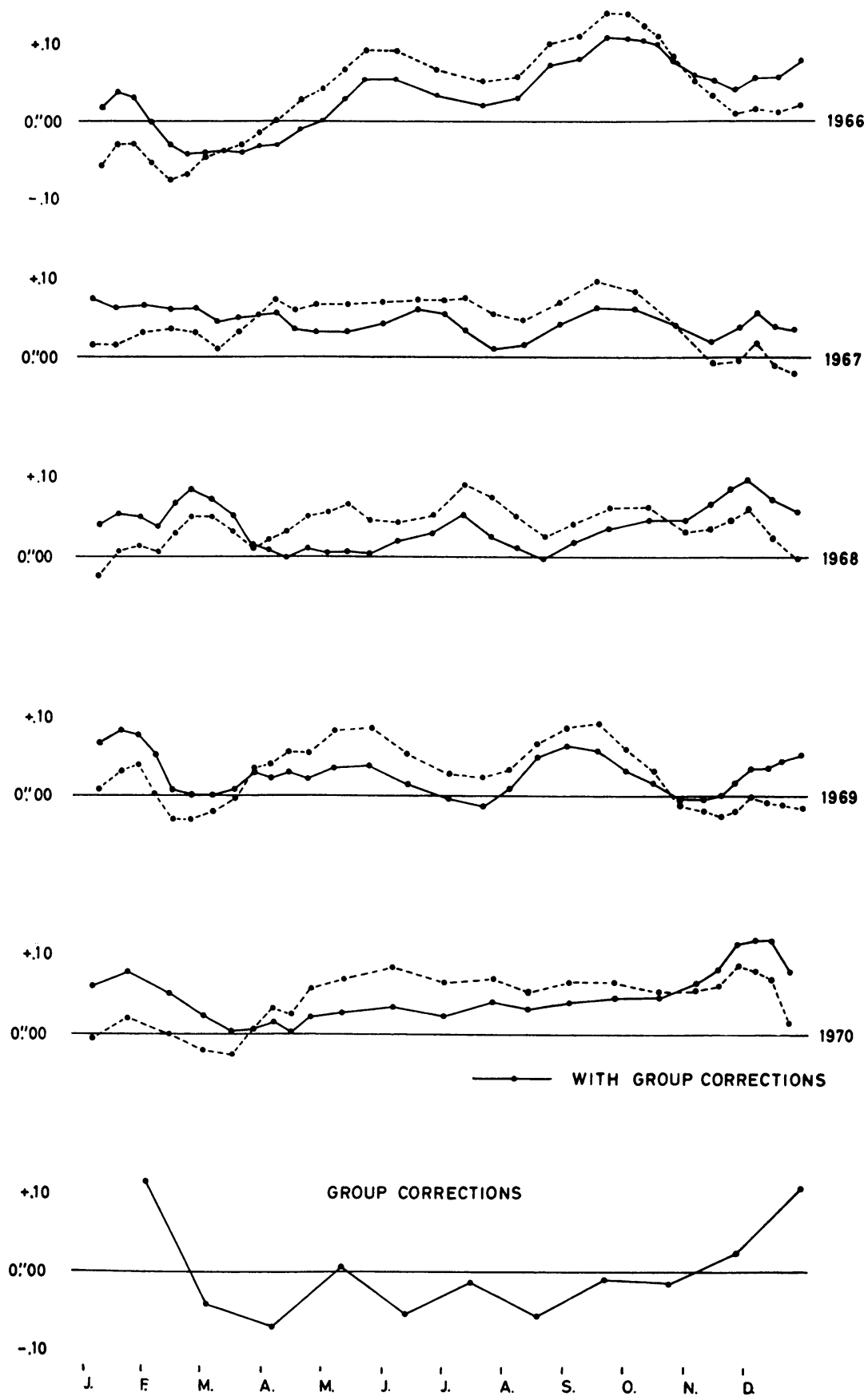

Fig. 1. Annual differences in latitude (Astrolabe - BIH). 
TABLE I

Group corrections in time and latitude. Astrolabe of Santiago

\begin{tabular}{|c|c|c|c|c|c|c|c|}
\hline \multirow[b]{2}{*}{ Gr. Nr. } & \multirow[b]{2}{*}{ R.A. } & \multirow[b]{2}{*}{1966} & \multicolumn{2}{|c|}{ Time (0\$̧001) } & \multicolumn{2}{|c|}{ Latitude (".01) } & \multirow[b]{2}{*}{$1969-70$} \\
\hline & & & $1967-68$ & $1969-70$ & 1966 & $1967-68$ & \\
\hline 1 & $0 . h ̣ 1$ & -2.4 & -2.1 & -6.3 & -0.9 & 0.0 & -5.3 \\
\hline 2 & 2.2 & 5.0 & 3.0 & 10.7 & -1.5 & -5.7 & -0.6 \\
\hline 3 & 4.4 & 2.8 & 7.1 & 6.0 & 2.5 & -0.6 & -2.6 \\
\hline 4 & 6.6 & 1.5 & 0.8 & 4.4 & 10.7 & 8.5 & 13.6 \\
\hline 5 & 8.7 & -0.8 & 3.2 & 3.9 & 11.6 & 11.0 & 9.4 \\
\hline 6 & 10.8 & -2.4 & -5.9 & -5.3 & -4.1 & -2.5 & -0.7 \\
\hline 7 & 13.0 & -1.4 & -0.9 & -3.1 & -7.0 & -8.1 & -5.3 \\
\hline 8 & 15.3 & 0.0 & -2.9 & -3.8 & 0.7 & 10.7 & 6.0 \\
\hline 9 & 17.4 & 5.2 & 4.8 & 8.6 & -5.3 & -5.4 & -6.4 \\
\hline 10 & 19.6 & -2.9 & -1.7 & -5.0 & -1.2 & 0.9 & -3.8 \\
\hline \multirow[t]{2}{*}{11} & 21.8 & -4.7 & -5.3 & -9.1 & -5.6 & -9.0 & -4.3 \\
\hline & C.E.D. & -10.5 & -29.3 & -0.7 & 12.1 & 41.2 & -2.9 \\
\hline
\end{tabular}

R.A.: Mean right ascension of the group.

C.E.D.: Closing error of the group differences.

The time differences, in units of arc and referred to the zenith of the observation point, are plotted with the applied group corrections in Figure 2. The most outstanding feature of these curves is the annual repetition of its general pattern. There is no significant difference between the curves with or without group corrections with respect to this annual effect.

At the present time we have no explanation for such an effect. It could be due to the influence of a large $\Delta \alpha_{\alpha}$ error in the FK4, not well represented by the group corrections. However, comparison of the group corrections in time with respect to an estimation of the $\Delta \alpha_{\alpha}$ error of the FK4, based on the observations made with a transit instrument at Cerro Calán, leads us to refuse this asumption. According to an agreement signed by the Soviet Academy of Sciences and Universidad de Chile for joint astrometric work between Pulkovo and Santiago Observatory in the Southern Hemisphere, observations of fundamental stars were carried out with a Zeiss Transit Instrument during 1963 and 1964 at Santiago (Loyola and Shishkina, 1968). The results in right ascension of these observations: Zeiss - FK4, for the zone $\delta=-21^{\circ}$ to $-45^{\circ}$ are given in Table II and Figure 3 (time), where it is possible to see that the curves: Astrolabe group corrections and Zeiss have a satisfactory conformity, although the Astrolabe and Zeiss zones are not strictly the same.

The possibility of an instrumental cause seems to us unlikely, if we consider the absence of a similar effect in latitude.

If we make some qualitative considerations about the site where our Astrolabe is installed, on the west border of a hill of about $100 \mathrm{~m}$ above the flat ground, close to very high mountains (Los Andes range) to the east covered with snow in winter in a 


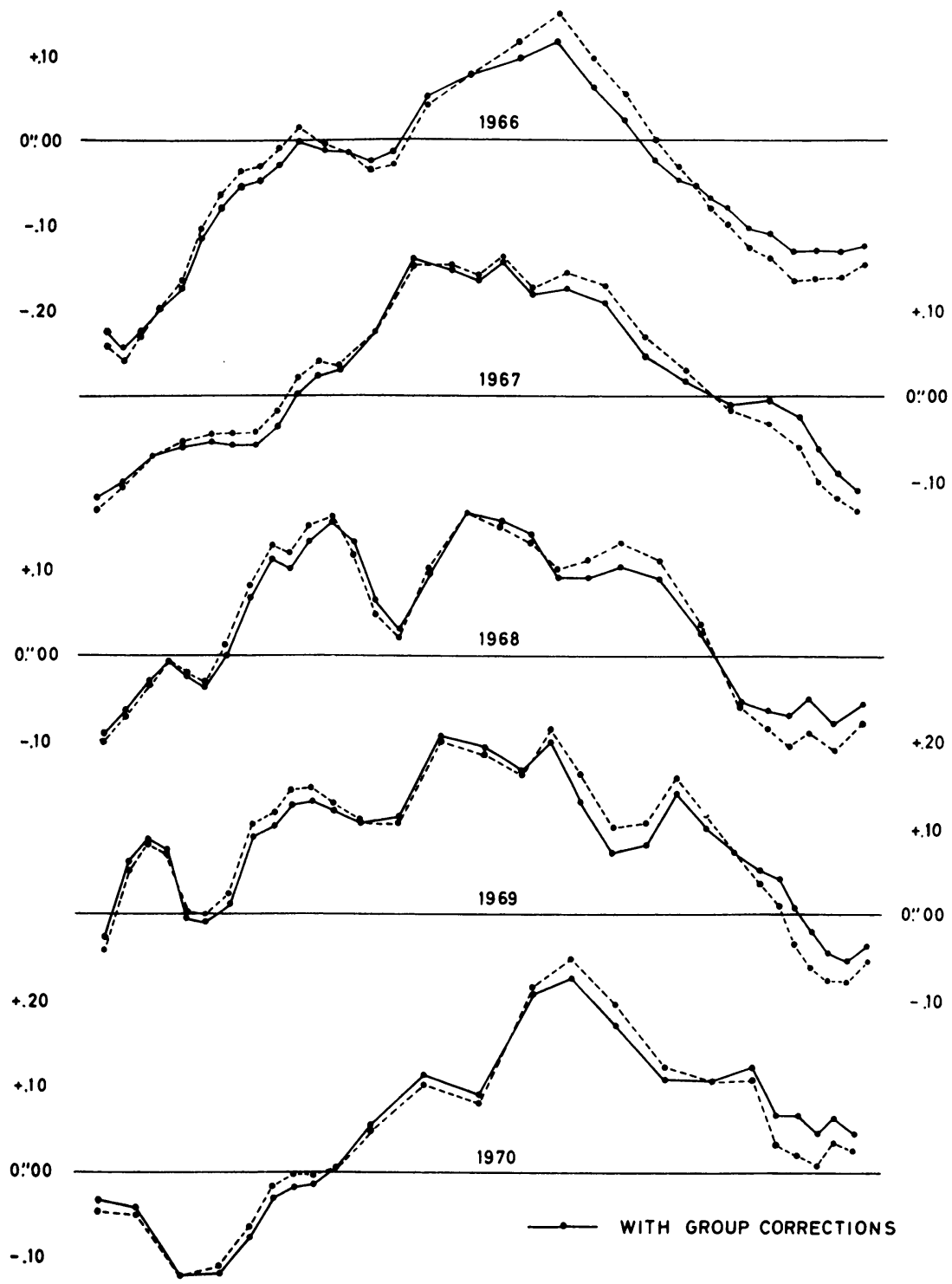

GROUP CORRECTIONS

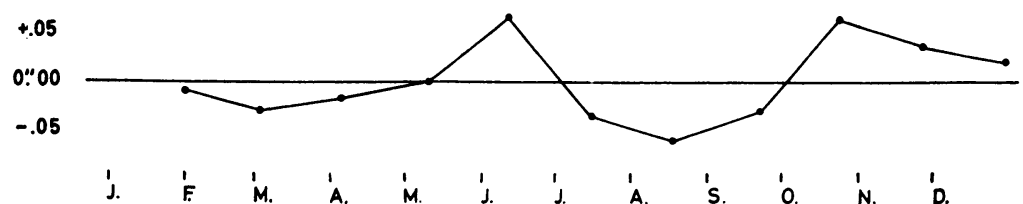

Fig. 2. Annual differences in time (Astrolabe - BIH). 
region with a seasonal wind system, one could expect a seasonal variation in a possible zenith refraction in the east-west direction. This cause seems to us as the most probable origin for the seasonal variation in time observed at Santiago. However, for the moment we have no additional data to solve this problem definitely.

TABLE II

Right ascension differences (Zeiss T.I.-FK4) (0s001)

\begin{tabular}{rrrr}
\hline$\alpha$ & \multicolumn{1}{c}{$\Delta \alpha$} & $\alpha$ & \multicolumn{1}{c}{$\Delta \alpha$} \\
\hline $0^{\mathrm{h}}$ & -3.5 & $12^{\mathrm{h}}$ & 1.3 \\
2 & -2.1 & 14 & 2.7 \\
4 & -1.1 & 16 & 3.8 \\
6 & 3.4 & 18 & 1.8 \\
8 & 0.5 & 20 & -1.8 \\
10 & 0.8 & 22 & -7.1
\end{tabular}

$+.15$

LATITUDE

10

.05

$0 . " 00$

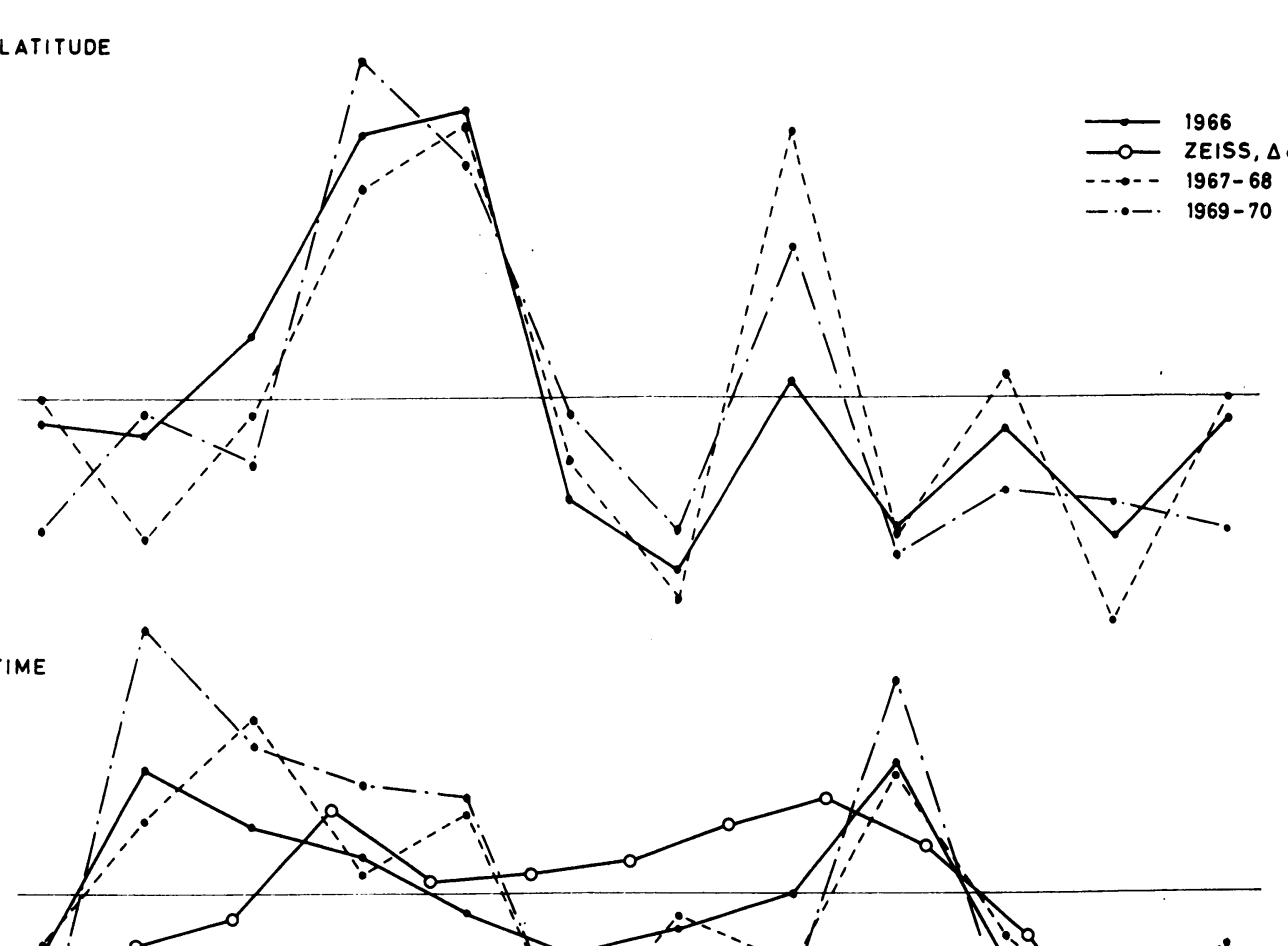

+.010 TIME

.005
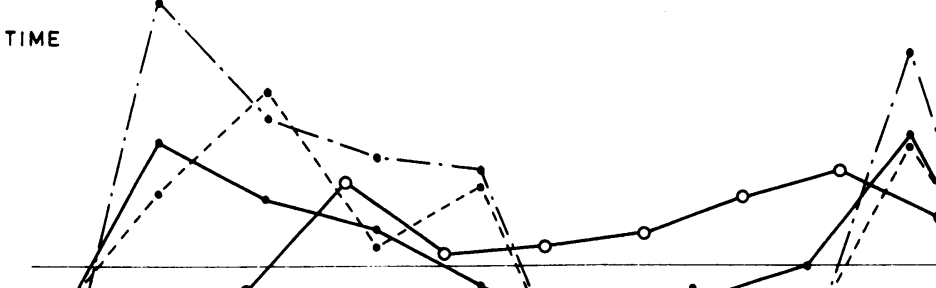

0.000

$-.005$
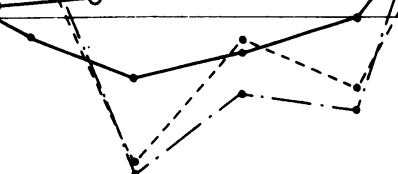

i

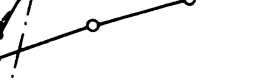

in
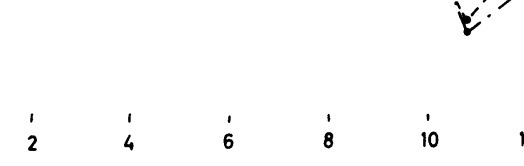

Fig. 3. Group corrections in latitude and time. 


\section{References}

Anguita, C. and Noël, F.: 1969, Astron. J. 74, 954.

Noël, F.: 1968; ESO Bull., No. 4, p. 9.

Loyola, P. and Shishkina, V. N.: 1968, Publ. Dept. Astron. Univ. Chile, Nr. 4, p. 55. 